\title{
Temperature dependence of the volume and surface contributions to the nuclear symmetry energy within the coherent density fluctuation model
}

\author{
A. N. Antonov, ${ }^{1}$ D. N. Kadrev, ${ }^{1}$ M. K. Gaidarov,${ }^{1, *}$ P. Sarriguren, ${ }^{2}$ and E. Moya de Guerra ${ }^{3}$ \\ ${ }^{1}$ Institute for Nuclear Research and Nuclear Energy, Bulgarian Academy of Sciences, Sofia 1784, Bulgaria \\ ${ }^{2}$ Instituto de Estructura de la Materia, IEM-CSIC, Serrano 123, E-28006 Madrid, Spain \\ ${ }^{3}$ Grupo de Física Nuclear, Departamento de Física Atómica, Molecular y Nuclear, Facultad de Ciencias Físicas, Unidad Asociada \\ UCM-CSIC(IEM), Universidad Complutense de Madrid, E-28040 Madrid, Spain
}

(Received 9 July 2018; revised manuscript received 15 October 2018; published 26 November 2018)

\begin{abstract}
The temperature dependence of the volume and surface components of the nuclear symmetry energy (NSE) and their ratio is investigated in the framework of the local density approximation. The results of these quantities for finite nuclei are obtained within the coherent density fluctuation model (CDFM). The CDFM weight function is obtained using the temperature-dependent proton and neutron densities calculated through the HFBTHO code, which solves the nuclear Skyrme-Hartree-Fock-Bogoliubov problem by using the cylindrical transformed deformed harmonic-oscillator basis. We present and discuss the values of the volume and surface contributions to the NSE and their ratio obtained for the $\mathrm{Ni}, \mathrm{Sn}$, and $\mathrm{Pb}$ isotopic chains around double-magic ${ }^{78} \mathrm{Ni},{ }^{132} \mathrm{Sn}$, and ${ }^{208} \mathrm{~Pb}$ nuclei. The results for the $T$ dependence of the considered quantities are compared with estimations made previously for zero temperature showing the behavior of the NSE components and their ratio, as well as with the available experimental data. The sensitivity of the results on various forms of the density dependence of the symmetry energy is studied. We confirm the existence of "kinks" in these quantities as functions of the mass number at $T=0 \mathrm{MeV}$ for the double closed-shell nuclei ${ }^{78} \mathrm{Ni}$ and ${ }^{132} \mathrm{Sn}$ and the lack of kinks for the $\mathrm{Pb}$ isotopes, as well as the disappearance of these kinks as the temperature increases.
\end{abstract}

DOI: 10.1103/PhysRevC.98.054315

\section{INTRODUCTION}

The study of the nuclear symmetry energy and, particularly, its density $(\rho)$ and temperature $(T)$ dependence is an important task in nuclear physics. This quantity is related to the energy connected with the conversion of isospin asymmetric nuclear matter into symmetric nuclear matter. It is an important ingredient of the nuclear equation of state in a wide range of densities and temperatures (see, e.g., [1-3]). Using approaches like the local density approximation [4-7] and the coherent density fluctuation model [8-10], knowledge of the equation of state can give information on the properties of finite systems. As noted in [11] information on the nuclear symmetry energy (NSE) from laboratory experiments can be obtained from quantities that are sensitive to it, such as static properties, nuclear excitations, collective motions, and heavy-ion reactions. One can add also interesting phenomena including supernova explosions, properties of neutron stars and rare isotopes, and frequencies and strain amplitudes of gravitational waves from both isolated pulsars and collisions involving neutron stars that depend strongly on the equation of state of neutron-rich nuclear matter. Here we would like to mention a broad range of works devoted to the study of $T$ dependence of single-particle properties of nuclear as well as neutron matter (e.g., [4,12-19]) and of the $\rho$ dependence of the NSE (e.g., [20-24]). Among the important studies

*gaidarov@inrne.bas.bg of the $T$ dependence of hot finite nuclear systems we note Ref. [25], on thermal Hartree-Fock (HF) calculations, the semiclassical approaches based on the microscopic SkyrmeHF formalism [26], the Thomas-Fermi (TF) approximation [27], the inclusion of continuum effects in HF calculations at finite temperature [28], the refined TF approach [29], and the extended TF method [30]. We mention also the Hartree-FockBogoliubov (HFB) models [31-34], as well as the relativistic TF approach with different relativistic mean-field nuclear forces [15]. Here we should also note the studies of the role of short-range and tensor nucleon-nucleon correlations, which change considerably the kinetic and potential energy contributions to the NSE (e.g., [1,11]).

The method of the coherent density fluctuation model (CDFM) [35,36] allowed us to make the transition from nuclear matter to finite nuclei in studies of the NSE for spherical [8] and deformed [9] nuclei, as well as for $\mathrm{Mg}$ isotopes [10] using the Brueckner energy-density functional (EDF) of asymmetric nuclear matter [37,38].

In our previous work [39] we used a similar method to investigate the $T$ dependence of the NSE for isotopic chains of even-even $\mathrm{Ni}, \mathrm{Sn}$, and $\mathrm{Pb}$ nuclei following the local density approximation [4-7] and using, instead of the Brueckner EDF, the Skyrme EDF with $\mathrm{SkM}^{*}$ and SLy4 forces. The $T$ dependent local densities $\rho(r, T)$ and kinetic energy densities $\tau(r, T)$ were calculated within a self-consistent Skyrme HFB method using the cylindrical transformed deformed harmonicoscillator basis (HFBTHO) [40,41] with the same forces. For comparison, the kinetic energy density $\tau$ was calculated also 
by the TF expression (for low temperatures) up to the $T^{2}$ term [16]. In addition, we studied the $T$ dependence of NSE for ${ }^{208} \mathrm{~Pb}$ using the extended TF method $[26,30]$ and the rigorous density functional approach [42] to obtain $T$-dependent local density distributions.

In his pioneering work [43] Feenberg in 1947 showed that the surface energy should contain a symmetry energy contribution due to the failure of nuclear saturation at the edge of the nucleus and that the volume saturation energy also has a symmetry energy term. In later works [44-47] analyses of the volume and surface components of the NSE, as well as of their ratio, were given. We should also mention some works in which the volume and surface components of the NSE and their ratio are explicitly related to the neutronskin thickness [48-58]. Agrawal et al. [4] pointed out the substantial change in NSE coefficients for finite nuclei with the temperature in comparison with the case of nuclear matter. Lee and Mekjian [16] emphasized the greater sensitivity of the surface component of the NSE to the temperature with respect to the volume energy term. We note also studies of the volume and surface NSE, e.g., in Refs. [59-65], including those analyzing the experimental nuclear binding energies $[64,65]$.

The values of the temperature-dependent symmetry energy coefficients for 69 spherical and nonspherical nuclei with mass $36 \leqslant A \leqslant 218$ and charge $14 \leqslant Z \leqslant 92$ numbers, respectively, have been evaluated in Ref. [66] in the subtracted finite-temperature TF framework using two different EDFs. A substantial temperature dependence of the surface symmetry energy was found, while the volume symmetry energy turned out to be less sensitive to the temperature. As a result, the symmetry energy coefficient of finite nuclei decreases as the temperature rises [66], an observation that has been confirmed in Ref. [39]. A study of the decomposition of the NSE into spin and isospin components is carried out in Ref. [67] within the Brueckner-Hartree-Fock approximation with two- and three-body forces.

In our work [68] the volume and surface contributions to the NSE and their ratio were calculated within the CDFM using two EDFs, namely, the Brueckner [37,38] and Skyrme (see Ref. [69]) ones. The CDFM weight function was obtained by means of the proton and neutron densities obtained from the self-consistent deformed HF + BCS method with densitydependent Skyrme interactions. The obtained results in the cases of $\mathrm{Ni}, \mathrm{Sn}$, and $\mathrm{Pb}$ isotopic chains were compared with results of other theoretical methods and with those from other approaches which used experimental data on binding energies, excitation energies to isobaric analog states, and neutron-skin thicknesses and with results of other theoretical methods. We note that in [68] the obtained values of the volume and surface components of the NSE and their ratio concern the case at $T=0 \mathrm{MeV}$.

The aim of the present work is to evaluate the abovementioned quantities for temperatures different from 0 . The $T$-dependent local density distributions $\rho_{p}(r, T)$ and $\rho_{n}(r, T)$ computed by the HFBTHO code are used to calculate the $T$-dependent CDFM weight function. Such an investigation of the thermal evolution of the NSE components and their ratio for isotopes belonging to the $\mathrm{Ni}, \mathrm{Sn}$, and $\mathrm{Pb}$ chains around the double-magic nuclei will extend our previous analysis of these nuclei considering them as cold systems [68]. At the same time, the obtained results within the CDFM provide additional information on the thermal mapping of the volume and surface symmetry energies that has been poorly investigated till now (e.g., Ref. [66]). In addition, we study the sensitivity of the calculated $T$-dependent quantities to different available forms of the density dependence of the symmetry energy.

The structure of this paper is the following. In Sec. II we present the main relationships for the NSE and its volume and surface components depending on the temperature that we use in our study, as well as the CDFM formalism, which provides a way to calculate the mentioned quantities. Section III contains the numerical results and discussion. The main conclusions of the study are given in Sec. IV.

\section{THEORETICAL FORMALISM}

We start this section with the expression for the nuclear energy given in the droplet model, which is an extension of the Bethe-Weizsäcker liquid drop model, to incorporate the surface asymmetry. It can be written as $[59,70]$

$$
\begin{aligned}
E(A, Z)= & -B A+E_{S} A^{2 / 3}+S^{V} A \frac{(1-2 Z / A)^{2}}{1+S^{S} A^{-1 / 3} / S^{V}} \\
& +E_{C} \frac{Z^{2}}{A^{1 / 3}}+E_{\mathrm{dif}} \frac{Z^{2}}{A}+E_{e x} \frac{Z^{4 / 3}}{A^{1 / 3}} \\
& +a \Delta A^{-1 / 2} .
\end{aligned}
$$

In Eq. (1) $B \simeq 16 \mathrm{MeV}$ is the binding energy per particle of bulk symmetric matter at saturation. $E_{S}, E_{C}, E_{\text {dif }}$, and $E_{\mathrm{ex}}$ are coefficients that correspond to the surface energy of symmetric matter, the Coulomb energy of a uniformly charged sphere, the diffuseness correction, and the exchange correction to the Coulomb energy, respectively, while the last term gives the pairing corrections ( $\Delta$ is a constant and $a=+1$ for odd-odd, 0 for odd-even, and -1 for even-even nuclei). $S^{V}$ is the volume symmetry energy parameter and $S^{S}$ is the modified surface symmetry energy parameter in the liquid model (see Ref. [59], where it is defined by $S^{S *}$ ).

In our previous work [39] we studied the temperature dependence of the NSE $S(T)$. For the aims of the present work we rewrite the symmetry energy [the third term on the right-hand side of Eq. (1)] in the form

$$
S(T) \frac{(N-Z)^{2}}{A},
$$

where

$$
S(T)=\frac{S^{V}(T)}{1+\frac{S^{S}(T)}{S^{V}(T)} A^{-1 / 3}}=\frac{S^{V}(T)}{1+A^{-1 / 3} / \kappa(T)}
$$

with

$$
\kappa(T) \equiv \frac{S^{V}(T)}{S^{S}(T)} .
$$

In the case of nuclear matter, where $A \longrightarrow \infty$ and $S^{S} / S^{V} \longrightarrow 0$, we have $S(T)=S^{V}(T)$. Also, at large $A$ Eq. (3) can be written in the known form (see Ref. [45])

$$
S(T)=\frac{S^{V}(T)}{1+\frac{S^{S}(T)}{S^{V}(T)} A^{-1 / 3}} \simeq c_{3}-\frac{c_{4}}{A^{1 / 3}},
$$


where $c_{3}=S^{V}$ and $c_{4}=S^{S}$. From Eq. (3) follow the relations of $S^{V}(T)$ and $S^{S}(T)$ with $S(T)$ :

$$
\begin{aligned}
& S^{V}(T)=S(T)\left(1+\frac{1}{\kappa(T) A^{1 / 3}}\right), \\
& S^{S}(T)=\frac{S(T)}{\kappa(T)}\left(1+\frac{1}{\kappa(T) A^{1 / 3}}\right) .
\end{aligned}
$$

In what follows we use essentially the CDFM scheme to calculate the NSE and its components (see Refs. [35,36,38]), in which the one-body density matrix $\rho\left(\mathbf{r}, \mathbf{r}^{\prime}\right)$ is a coherent superposition of the one-body density matrices $\rho_{x}\left(\mathbf{r}, \mathbf{r}^{\prime}\right)$ for spherical "pieces" of nuclear matter ("fluctons") with densities $\rho_{x}(\mathbf{r})=\rho_{0}(x) \Theta(x-|\mathbf{r}|)$ and $\rho_{0}(x)=3 A / 4 \pi x^{3}$. It has the form

$$
\rho\left(\mathbf{r}, \mathbf{r}^{\prime}\right)=\int_{0}^{\infty} d x|\mathcal{F}(x)|^{2} \rho_{x}\left(\mathbf{r}, \mathbf{r}^{\prime}\right)
$$

with

$\rho_{x}\left(\mathbf{r}, \mathbf{r}^{\prime}\right)=3 \rho_{0}(x) \frac{j_{1}\left(k_{F}(x)\left|\mathbf{r}-\mathbf{r}^{\prime}\right|\right)}{\left(k_{F}(x)\left|\mathbf{r}-\mathbf{r}^{\prime}\right|\right)} \Theta\left(x-\frac{\left|\mathbf{r}+\mathbf{r}^{\prime}\right|}{2}\right)$,

where $j_{1}$ is the first-order spherical Bessel function and

$$
k_{F}(x)=\left(\frac{3 \pi^{2}}{2} \rho_{0}(x)\right)^{1 / 3} \equiv \frac{\beta}{x}
$$

with

$$
\beta=\left(\frac{9 \pi A}{8}\right)^{1 / 3} \simeq 1.52 A^{1 / 3}
$$

is the Fermi momentum of the nucleons in the flucton with radius $x$. The density distribution in the CDFM has the form

$$
\rho(\mathbf{r})=\int_{0}^{\infty} d x|\mathcal{F}(x)|^{2} \rho_{0}(x) \Theta(x-|\mathbf{r}|)
$$

It follows from (12) that in the case of a monotonically decreasing local density $(d \rho / d r \leqslant 0)$ the weight function $|\mathcal{F}(x)|^{2}$ can be obtained from a known density (theoretically or experimentally obtained):

$$
|\mathcal{F}(x)|^{2}=-\left.\frac{1}{\rho_{0}(x)} \frac{d \rho(r)}{d r}\right|_{r=x} .
$$

We have shown in our previous works $[8,9,68]$ that the NSE in the CDFM for temperature $T=0$ can be obtained in the form

$$
S=\int_{0}^{\infty} d x|\mathcal{F}(x)|^{2} S[\rho(x)],
$$

where the symmetry energy for the asymmetric nuclear matter, which depends on the density $S[\rho(x)]$, has to be determined using a chosen EDF (in [68] Brueckner and Skyrme EDFs are used).

In this work the $T$-dependent NSE $S(T)$ is calculated by expressions similar to Eq. (14) but containing $T$-dependent quantities:

$$
S(T)=\int_{0}^{\infty} d x|\mathcal{F}(x, T)|^{2} S[\rho(x, T)] .
$$

In Eq. (15) the weight function $|\mathcal{F}(x, T)|^{2}$ depends on the temperature through the temperature-dependent total density
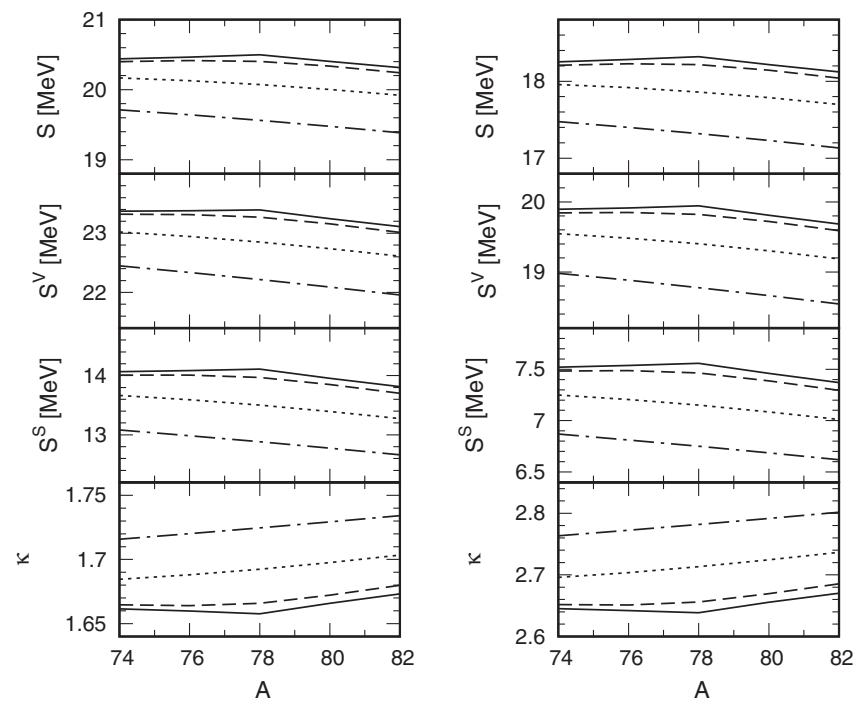

FIG. 1. Mass dependence of the NSE $S(T)$, its volume $S^{V}(T)$ and surface $S^{S}(T)$ components, and their ratio $\kappa(T)$ for nuclei from the Ni isotopic chain at temperatures $T=0 \mathrm{MeV}$ (solid line), $T=$ $1 \mathrm{MeV}$ (dashed line), $T=2 \mathrm{MeV}$ (dotted line), and $T=3 \mathrm{MeV}$ (dash-dotted line) calculated with the $\mathrm{SkM}^{*}$ Skyrme interaction for values of the parameter $\gamma=0.3$ (left panel) and $\gamma=0.4$ (right panel).

distribution $\rho_{\text {total }}(r, T)$,

$$
|\mathcal{F}(x, T)|^{2}=-\left.\frac{1}{\rho_{0}(x)} \frac{d \rho_{\text {total }}(r, T)}{d r}\right|_{r=x},
$$

where

$$
\rho_{\text {total }}(r, T)=\rho_{p}(r, T)+\rho_{n}(r, T),
$$

$\rho_{p}(r, T)$ and $\rho_{n}(r, T)$ being the proton and neutron $T$ dependent densities, which in our work [39] were calculated using the HFB method with the transformed harmonicoscillator basis and the HFBTHO code [40].

As mentioned, in the present work we consider the $T$ dependence of the NSE $S(T)$ but, also, of its volume $S^{V}(T)$ and surface $S^{S}(T)$ components and their ratio $\kappa(T)$ [Eq. (4)]. Following Refs. $[48,56,61,68]$ an approximate expression for the ratio $\kappa(T)$ can be written within the CDFM,

$$
\kappa(T)=\frac{3}{R \rho_{0}} \int_{0}^{\infty} d x|\mathcal{F}(x, T)|^{2} x \rho_{0}(x)\left\{\frac{S\left(\rho_{0}\right)}{S[\rho(x, T)]}-1\right\},
$$

where $|\mathcal{F}(x, T)|^{2}$ is determined by Eq. (16), $R=r_{0} A^{1 / 3}[61]$, and $S\left(\rho_{0}\right)$ is the NSE at equilibrium nuclear matter density $\rho_{0}$ and $T=0 \mathrm{MeV}$. For instance, the values of $S\left(\rho_{0}\right)$ for different Skyrme forces in the Skyrme EDF are listed in Table II in Ref. [68]. In what follows we use the commonly employed power parametrization (first, in Sec. III A) for the density dependence of the symmetry energy (e.g., $[49,56,61])$ :

$$
S[\rho(x, T)]=S^{V}(T)\left[\frac{\rho(x, T)}{\rho_{0}}\right]^{\gamma} .
$$

There exist various estimations for the value of the parameter $\gamma$. For instance, in Ref. [61] $\gamma=0.5 \pm 0.1$ and in Ref. [56] 

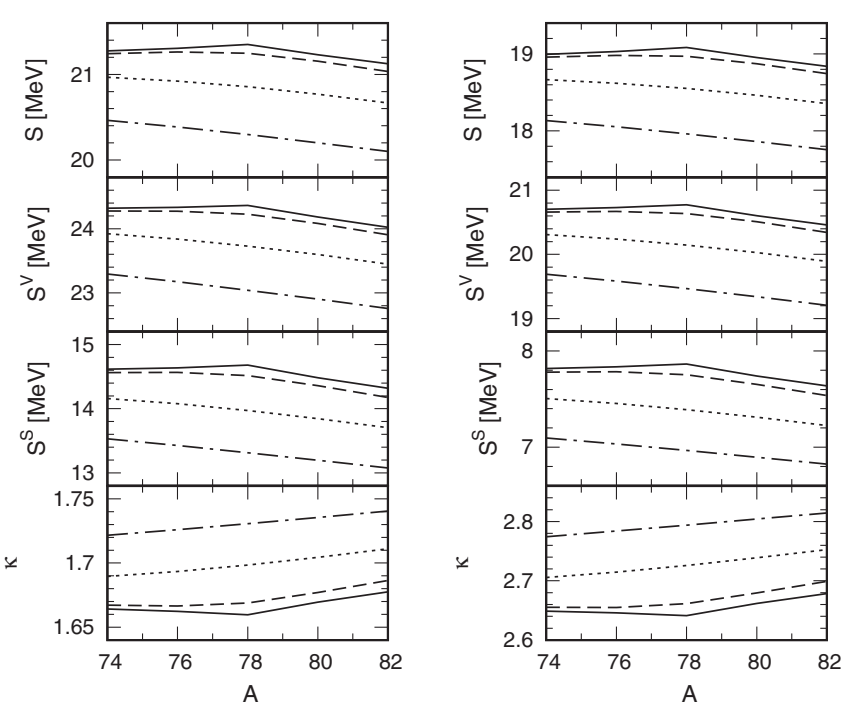

FIG. 2. Same as Fig. 1, but with the SLy4 Skyrme interaction.

$0.54 \leqslant \gamma \leqslant 0.77$. The estimations in Ref. [71] (listed in Table 2 there) of the NSE based on different cases within the chiral effective field theory and from other predictions are $\gamma=0.60 \pm 0.05\left(\mathrm{~N}^{2} \mathrm{LO}\right), \gamma=0.55 \pm 0.03\left(\mathrm{~N}^{3} \mathrm{LO}\right), \gamma=$ 0.55 (DBHF), and 0.79 (APR [72]). Another estimation of $\gamma=0.72 \pm 0.19$ is also given in Ref. [73].

Using Eq. (19) [and having in mind that $S\left(\rho_{0}\right)=S^{V}$ ], Eqs. (15) and (18) can be rewritten as follows:

$$
\begin{aligned}
& S(T)=S\left(\rho_{0}\right) \int_{0}^{\infty} d x|\mathcal{F}(x, T)|^{2}\left[\frac{\rho(x, T)}{\rho_{0}}\right]^{\gamma}, \\
& \kappa(T) \equiv \frac{S^{V}(T)}{S^{S}(T)}= \frac{3}{R \rho_{0}} \int_{0}^{\infty} d x|\mathcal{F}(x, T)|^{2} x \rho_{0}(x) \\
& \times\left\{\left[\frac{\rho_{0}}{\rho(x, T)}\right]^{\gamma}-1\right\} .
\end{aligned}
$$
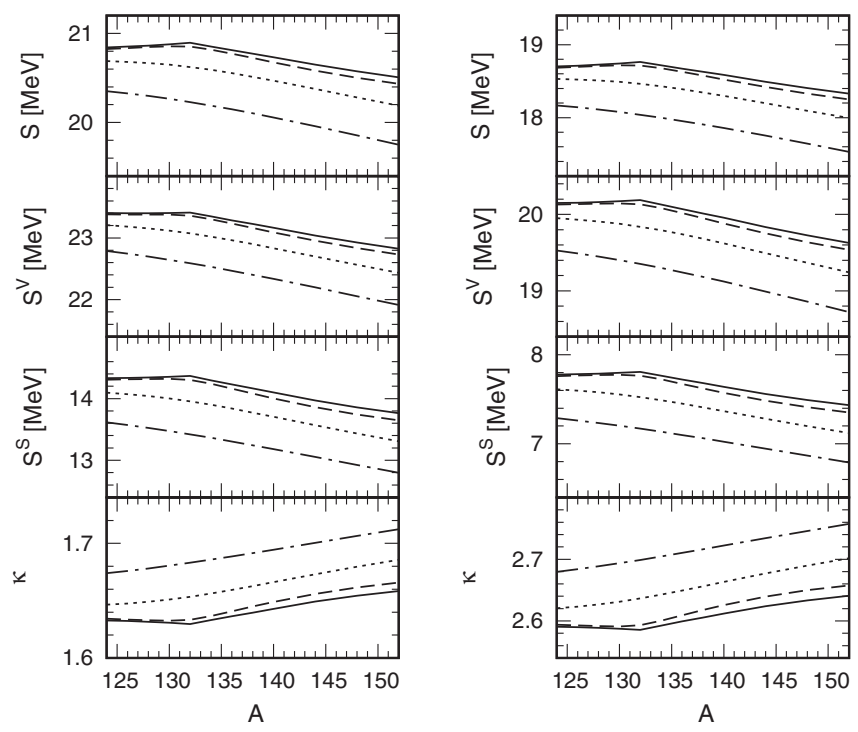

FIG. 3. Same as Fig. 1, but for nuclei from the Sn isotopic chain.
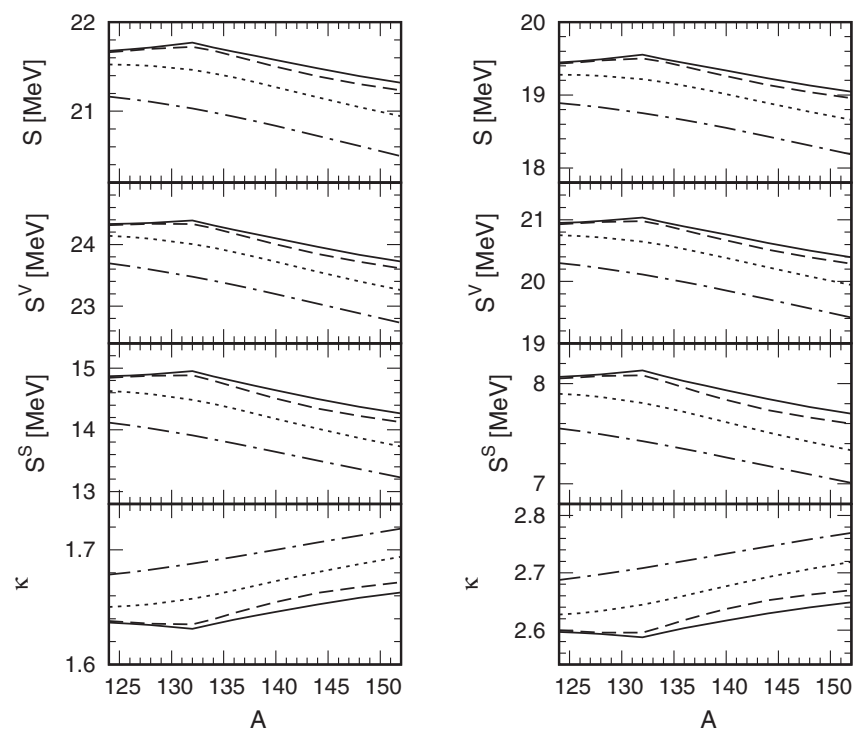

FIG. 4. Same as Fig. 2, but for nuclei from the $\mathrm{Sn}$ isotopic chain.

\section{RESULTS OF CALCULATIONS AND DISCUSSION}

\section{A. Results with the density dependence of the symmetry energy given by Eq. (19)}

Calculations of the $T$-dependent nuclear symmetry energy $S(T)$, its volume $S^{V}(T)$ and surface $S^{S}(T)$ components, and their ratio $\kappa(T)=S^{V}(T) / S^{S}(T)$ were performed using the relationships (15) and (18)-(21) with the weight function $|\mathcal{F}(x, T)|^{2}$ from Eqs. (16) and (17). As mentioned in Sec. II, the $T$-dependent proton $\rho_{p}(r, T)$ and neutron $\rho_{n}(r, T)$ density distributions, as well as the total density $\rho_{\text {total }}(r, T)$ [Eq. (17)], were calculated using the HFBTHO code from Ref. [40] with the Skyrme EDF for SkM* and SLy4 forces. We note that in the calculations of $S(T)$ [Eq. (20)] and $\kappa(T)$ [Eq. (21)] we use the weight function $|\mathcal{F}(x, T)|^{2}$ from Eq. (16), where the density distributions for finite nuclei are used. The quantity
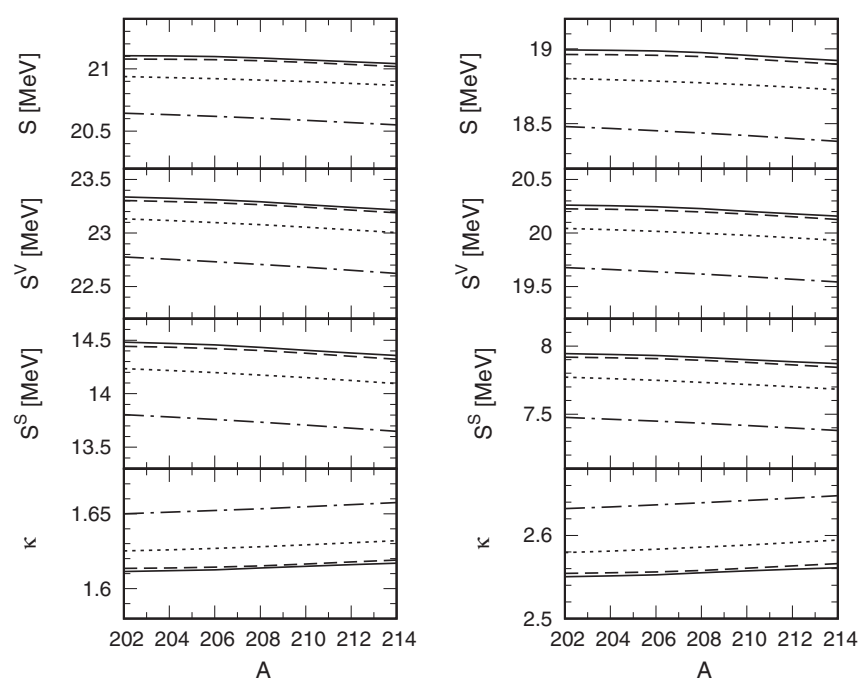

FIG. 5. Same as Fig. 1, but for nuclei from the $\mathrm{Pb}$ isotopic chain. 


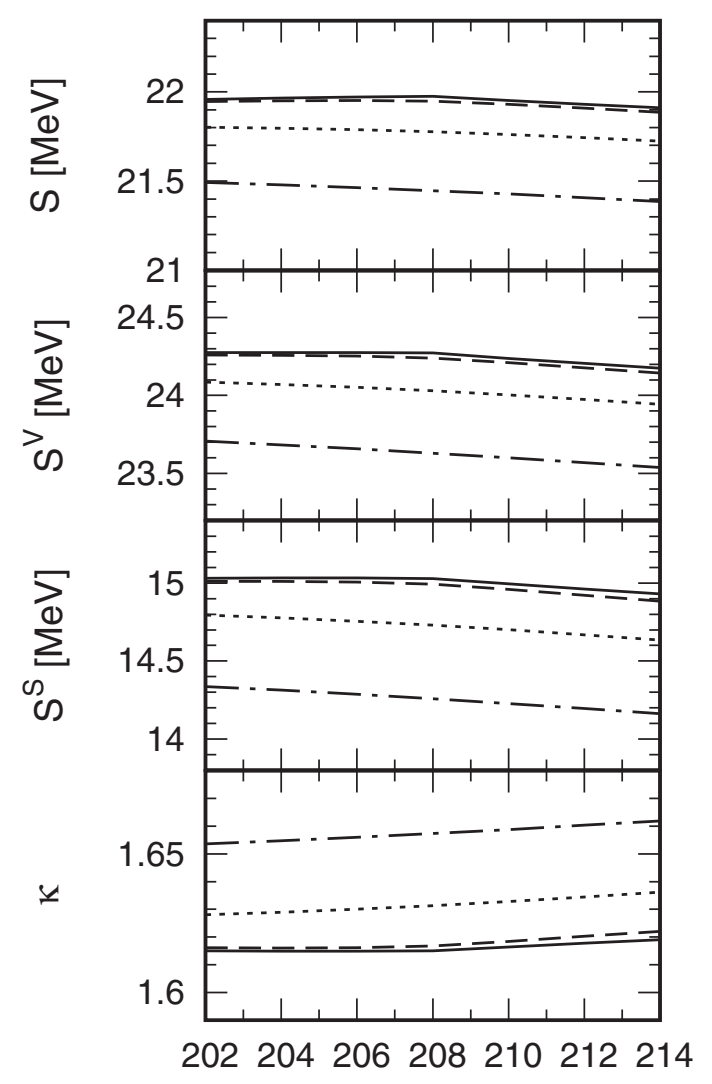

A

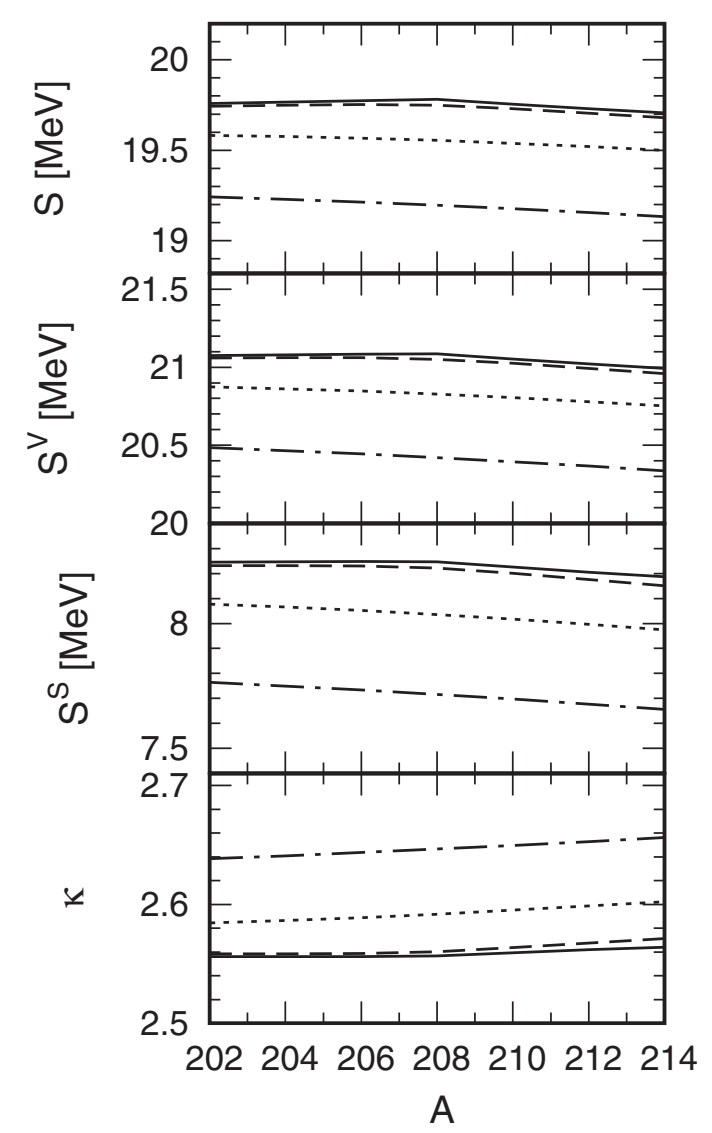

FIG. 6. Same as Fig. 2, but for nuclei from the $\mathrm{Pb}$ isotopic chain.
$S[\rho(x, T)]$ in Eqs. (15) and (18) is the symmetry energy for asymmetric nuclear matter chosen in the parametrized form of Eq. (19). Our calculations are performed for the $\mathrm{Ni}, \mathrm{Sn}$, and $\mathrm{Pb}$ isotopic chains.

Studying the $T$ dependence of the mentioned quantities we observed (as shown below) a certain sensitivity of the results to the value of the parameter $\gamma$ in Eq. (19). In order to make the choice of its value we imposed the following physical conditions: (i) the obtained results for the considered quantities at $T=0 \mathrm{MeV}$ must be equal or close to those obtained for the same quantities in our previous works on the NSE, its components, and their ratio [39,68]; and (ii) their values for $T=0 \mathrm{MeV}$ must be compatible with the available experimental data (see, e.g., the corresponding references in [68]).

In Figs. 1-6 the results for $S(T), S^{V}(T), S^{S}(T)$, and $\kappa=$ $S^{V}(T) / S^{S}(T)$ are given as functions of the mass number $A$ for the isotopic chains of $\mathrm{Ni}, \mathrm{Sn}$, and $\mathrm{Pb}$ nuclei for temperatures $T=0-3 \mathrm{MeV}$ calculated using the SkM* and SLy4 Skyrme forces. The results are presented for two values of the parameter $\gamma=0.3$ and 0.4 . The reason for this choice is related to the physical criterion mentioned above. It can be seen that at $T=0 \mathrm{MeV}$ and $\gamma=0.4$ the value of $\kappa$ is around 2.6. This result is in agreement with our previous result obtained in the case of the Brueckner EDF in Ref. [68], namely, $2.10 \leqslant$ $\kappa \leqslant 2.90$. The latter is compatible with the published values of $\kappa$ extracted from nuclear properties presented in Ref. [61] from isobaric analog states and skins [49] $(2.6 \leqslant \kappa \leqslant 3.0)$ and from masses and skins [48] $(2.0 \leqslant \kappa \leqslant 2.8)$. In the case of $\gamma=0.3$ our result for $T=0 \mathrm{MeV}$ is $\kappa=1.65$, which is in agreement with the analyses of data in Ref. [61] (1.6 $\leqslant$ $\kappa \leqslant 2.0$ ), as well as with the results of our work [68] in the case of the Skyrme EDF, namely, for the Ni isotopic chain $1.5 \leqslant \kappa \leqslant 1.7$, for the $\mathrm{Sn}$ isotopic chain $1.52 \leqslant \kappa \leqslant 2.1$, and for the $\mathrm{Pb}$ isotopic chain $1.65 \leqslant \kappa \leqslant 1.75$, all obtained with SLy4 and SGII forces.

Before comparing our results for $S(T)$ at $T=0 \mathrm{MeV}$ with our previous results from Ref. [39] (there the NSE is denoted $\left.e_{\text {sym }}\right)$, we mention that though the latter are in good agreement with theoretical predictions for some specific nuclei reported by other authors, we showed that they depend on the suggested definitions of this quantity. The comparison of the results in the present work for $S$ at $T=0 \mathrm{MeV}$ with those from our work in Ref. [39] (the latter illustrated in Fig. 12 there) shows that they agree with our present values of $S$ within the range of $\gamma=0.3-0.4$, except in the case of $\mathrm{Pb}$ with $\mathrm{SLy} 4$ force, where the present results are somewhat lower.

It can be seen in Figs. 1-6 that the quantities $S(T)$, $S^{V}(T)$, and $S^{S}(T)$ decrease with increasing temperatures $(T=0-3 \mathrm{MeV})$, while $\kappa(T)$ slowly increases when $T$ increases. This is true both for Skyrme forces (SkM* and SLy4) and for the three isotopic chains of the $\mathrm{Ni}, \mathrm{Sn}$, and $\mathrm{Pb}$ nuclei. Here we would like to note that the values of $\gamma$ between 0.3 and 0.4 , which give agreement of the studied quantities with the data, as well as with our previous results for $T=0 \mathrm{MeV}$, are in the lower part of the estimated limits of the values of $\gamma$ (e.g., in the case of $\gamma=0.5 \pm 0.1$ [61]). 

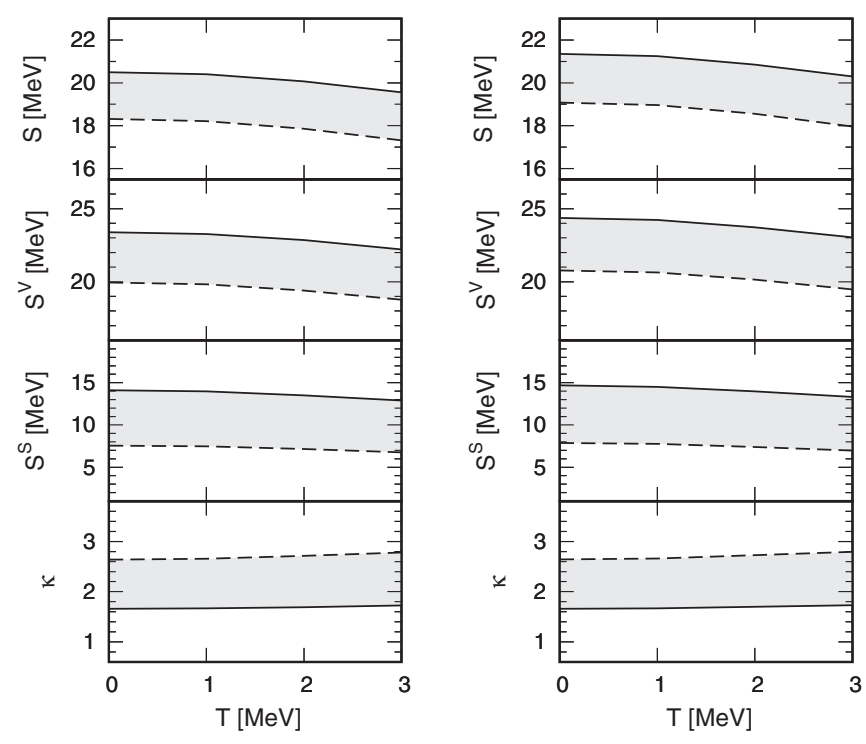

FIG. 7. Temperature dependence of the NSE $S(T)$, its volume $S^{V}(T)$ and surface $S^{S}(T)$ components, and their ratio $\kappa(T)$ obtained for values of the parameter $\gamma=0.3$ (solid line) and $\gamma=0.4$ (dashed line) with $\mathrm{SkM}^{*}$ (left panel) and SLy4 (right panel) forces for ${ }^{78} \mathrm{Ni}$ nucleus.

It can be seen also in Figs. 1-6 that there are "kinks" in the curves of $S(T), S^{V}(T), S^{S}(T)$, and $\kappa(T)$ for $T=0 \mathrm{MeV}$ in the cases of the double closed-shell nuclei ${ }^{78} \mathrm{Ni}$ and ${ }^{132} \mathrm{Sn}$ but no kinks in the $\mathrm{Pb}$ chain. This had been observed also in our previous work on $S(T)$ [39], as well as on its volume and surface components and their ratio $\kappa$ at $T=0 \mathrm{MeV}$ in Ref. [68].

In Figs. 7-9 we give the results for the $T$ dependence of $S(T), S^{V}(T), S^{S}(T)$, and $\kappa(T)$ for the double-magic ${ }^{78} \mathrm{Ni}$, ${ }^{132} \mathrm{Sn}$, and ${ }^{208} \mathrm{~Pb}$ nuclei obtained using both $\mathrm{SkM}^{*}$ and SLy4 Skyrme forces. The results are represented by gray areas between the curves for the values of the parameter $\gamma=0.3$
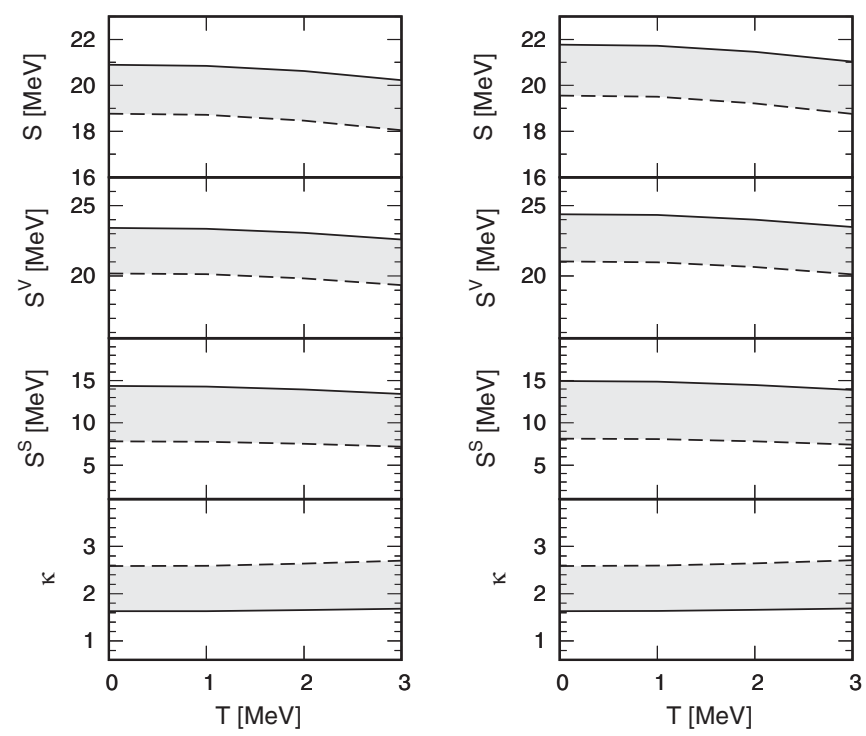

FIG. 8. Same as Fig. 7, but for ${ }^{132}$ Sn nucleus. and $\gamma=0.4$. It can be seen that $S(T), S^{V}(T)$, and $S^{S}(T)$ decrease, while $\kappa(T)$ slowly increases, with the increase in temperature for both Skyrme forces.

\section{B. Comparison with results from alternative parametrizations of the density dependence of the symmetry energy}

In this part of our work we study the sensitivity of the obtained results to different density dependences of the symmetry energy. First, we note Eq. (6) from Ref. [74]:

$$
S(\rho)=C_{k}\left(\frac{\rho}{\rho_{0}}\right)^{2 / 3}+C_{1}\left(\frac{\rho}{\rho_{0}}\right)+C_{2}\left(\frac{\rho}{\rho_{0}}\right)^{1.52} .
$$

This relationship, which coincides with the shape from the density-dependent M3Y interaction [75], is similar to that of Eq. (9) in Ref. [76], where the last term of Eq. (22) is exchanged for $C_{2}\left(\rho / \rho_{0}\right)^{5 / 3}$. Second, we use also the dependence of the symmetry energy $[54,74]$

$$
S(\rho)=12.5\left(\frac{\rho}{\rho_{0}}\right)^{2 / 3}+17.6\left(\frac{\rho}{\rho_{0}}\right)^{\gamma} .
$$

It is noted in [54] that the analysis of both isospin diffusion and double ratio data involving neutron and proton spectra by an improved quantum molecular dynamics transport model suggests values of $\gamma=0.4-1.05$.

Concerning the values of the coefficients $C_{1}$ and $C_{2}$ in Eq. (22), we determined them by fitting the three curves of $S(\rho)$ [for different values of $S\left(\rho_{0}\right)$ ] presented in Fig. 4 of Ref. [74] and note them as $S_{1}(\rho)$ when $S\left(\rho_{0}\right)=29.4 \mathrm{MeV}$, $S_{2}(\rho)$ when $S\left(\rho_{0}\right)=31.6 \mathrm{MeV}$, and $S_{3}(\rho)$ when $S\left(\rho_{0}\right)=$ $33.8 \mathrm{MeV}$. We mention also the remark in Ref. [74] that the expressions for $S(\rho)$ in Eqs. (19) and (23) "do not reproduce the density dependence of the symmetry energy as predicted by the mean-field approach around nuclear saturation density." Nevertheless, in what follows compare our results from Sec. III A with those calculated using not only Eq. (22) but also $S(\rho)$ from Eq. (23), which we note as $S_{4}(\rho)$.

Figure 10 illustrates the behavior of the density dependence of the symmetry energy $S(\rho)$ by giving some of the curves for different functions, namely, $S_{2}(\rho)$, three curves using Eq. (19), which we label as $S_{0}(\rho)$ with $\gamma=0.3,0.4$, and 0.7 , as well as three curves for $S_{4}(\rho)$, which correspond to $\gamma=0.2,0.3$, and 0.7 . At this point we emphasize that the CDFM weight function $|\mathcal{F}(x, T)|^{2}$ which is used in Eqs. (15) and (18) has the form of a bell with a maximum around $x=$ $R_{1 / 2}$ at which the value of the density $\rho\left(x=R_{1 / 2}\right)$ is half the value of the central density equal to $\rho_{0}\left[\rho\left(R_{1 / 2}\right) / \rho_{0}=0.5\right]$. So, namely, in this region (around $\rho / \rho_{0}=0.5$ ), the values of the different $S(\rho)$ 's play the main role in the calculations of $S(T)$ [Eq. (15)] and $\kappa(T)$ [Eq. (18)].

In Fig. 11 we consider, as an example, the mass dependence of $S(T), S^{V}(T), S^{S}(T)$, and $\kappa=S^{V}(T) / S^{S}(T)$ in the case of the Ni isotopic chain for temperatures $T=0-3 \mathrm{MeV}$ using the SLy4 Skyrme force. The results are given when the symmetry energy has the form of $S_{4}(\rho)$ at $\gamma=0.2$ and 0.3 . One can see that, e.g., the value of $\kappa$ at $T=0 \mathrm{MeV}$ and $\gamma=0.2$ is 1.90 , which is close to the result obtained using Eqs. (19) and (21) for $\gamma=0.3$ (it is $\kappa=1.66$ ), shown in the left panel in Fig. 2. The value of $\kappa$ when $\gamma=0.3$ is 2.69, 

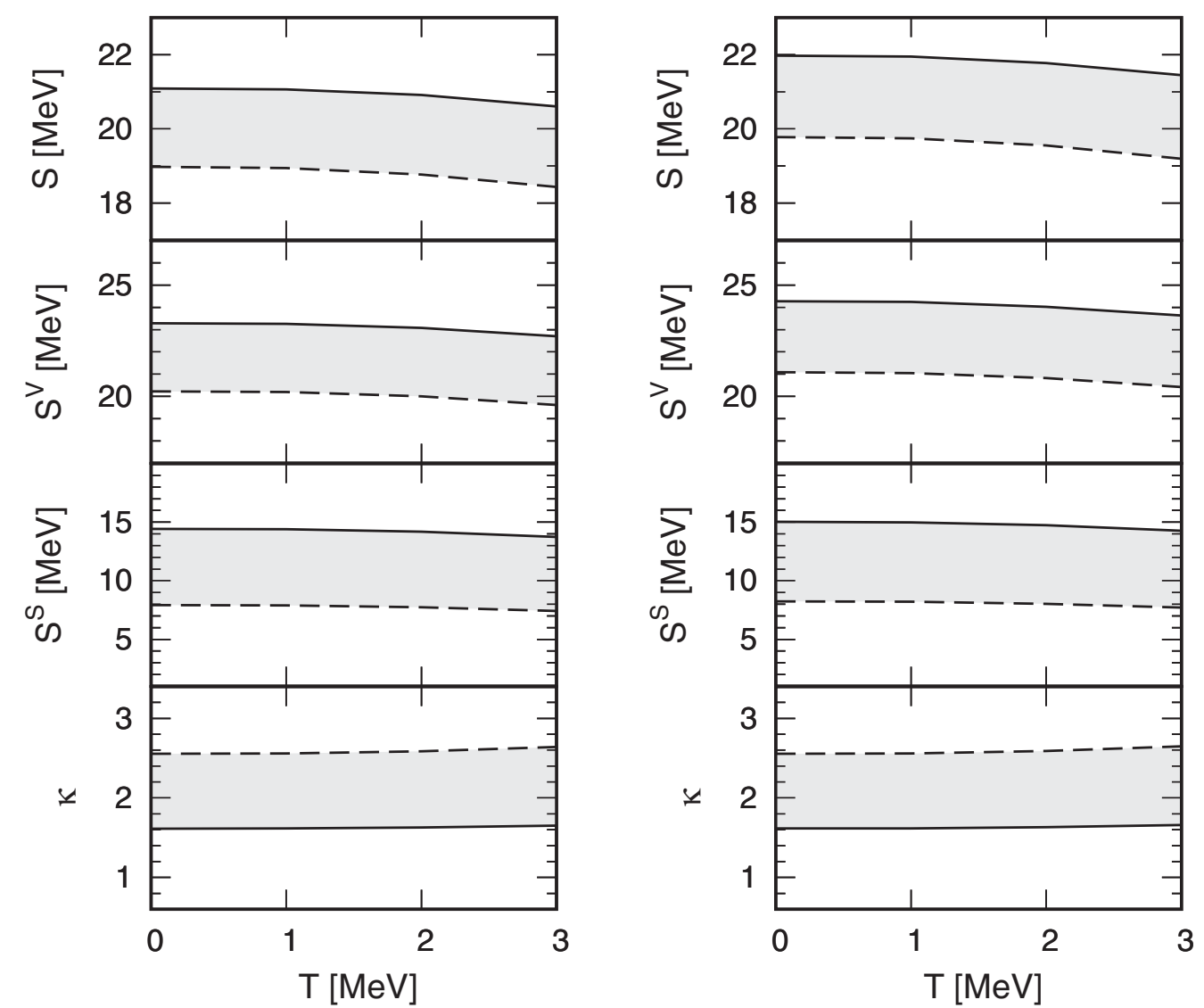

FIG. 9. Same as Fig. 7, but for ${ }^{208} \mathrm{~Pb}$ nucleus.

which is similar to that in the case of Eqs. (19) and (21) for $\gamma=0.4(\kappa=2.64)$, shown in the right panel in Fig. 2 . There exist in these cases similarities also to the behavior of the quantities $S(T), S^{V}(T)$, and $S^{S}(T)$ as functions of

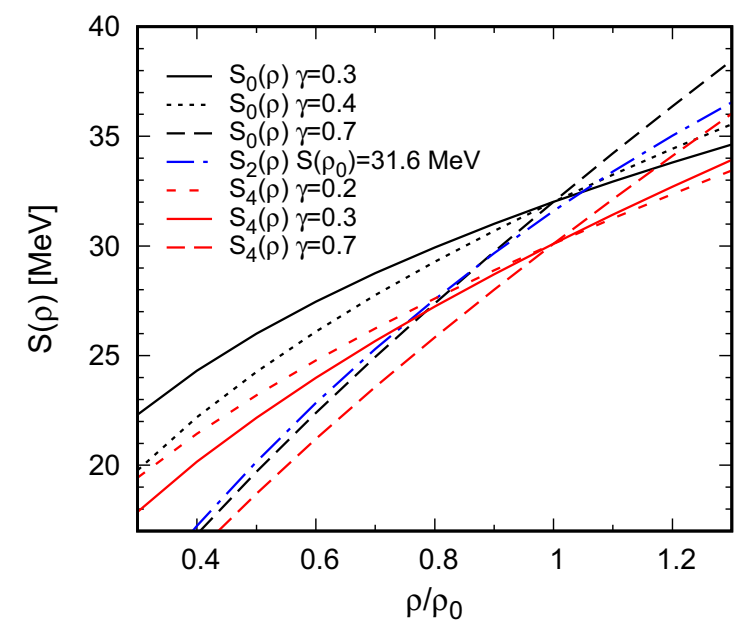

FIG. 10. Behavior of the density-dependent symmetry energy: $S_{2}(\rho)$ with $S\left(\rho_{0}\right)=31.6 \mathrm{MeV}$ [Eq. (22) with $C_{k}=17.47, C_{1}=$ 27.94, and $C_{2}=-13.81$; dash-dotted blue line]; $S_{4}(\rho)$ [Eq. (23)] with $\gamma=0.2$ (short-dashed red line), $\gamma=0.3$ (solid red line), $\gamma=$ 0.7 (dashed red line), and $S_{0}(\rho)$ [Eq. (19)] with $\gamma=0.3$ (solid black line), $\gamma=0.4$ (dotted black line), and $\gamma=0.7$ (dashed black line).
$T$ in the corresponding cases. The reason for the mentioned similarities is the closeness of the corresponding curves shown in Fig. 10 in the region around $\rho / \rho_{0}=0.5$.

In Fig. 12 we show, as an example, the $T$ dependence of $S(T), S^{V}(T), S^{S}(T)$, and $\kappa$ in the case of the ${ }^{78} \mathrm{Ni}$ nucleus
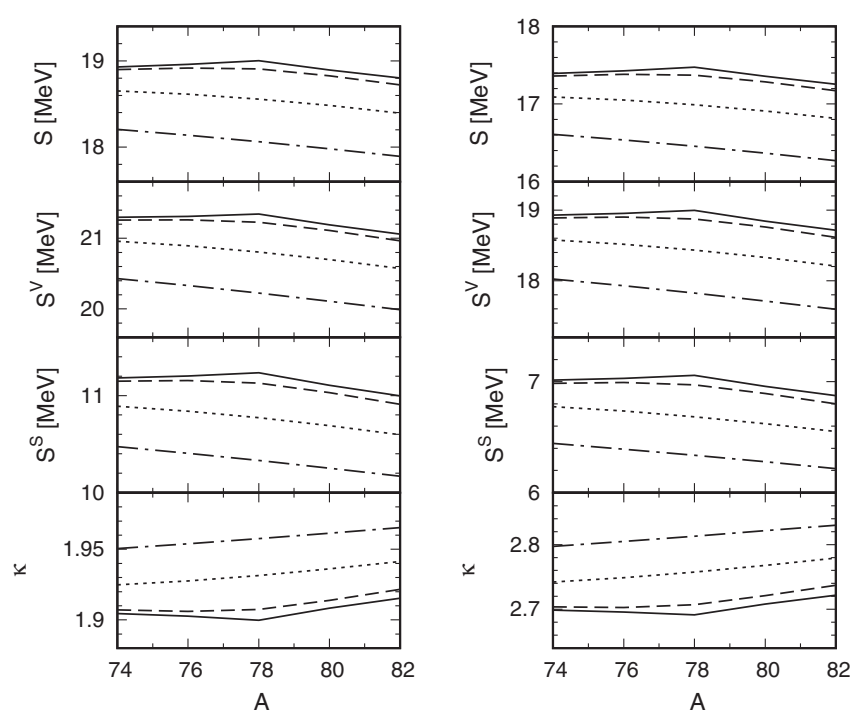

FIG. 11. Same as Fig. 2, but with the use of $S_{4}(\rho)$ [Eq. (23)] for values of the parameter $\gamma=0.2$ (left panel) and $\gamma=0.3$ (right panel). 


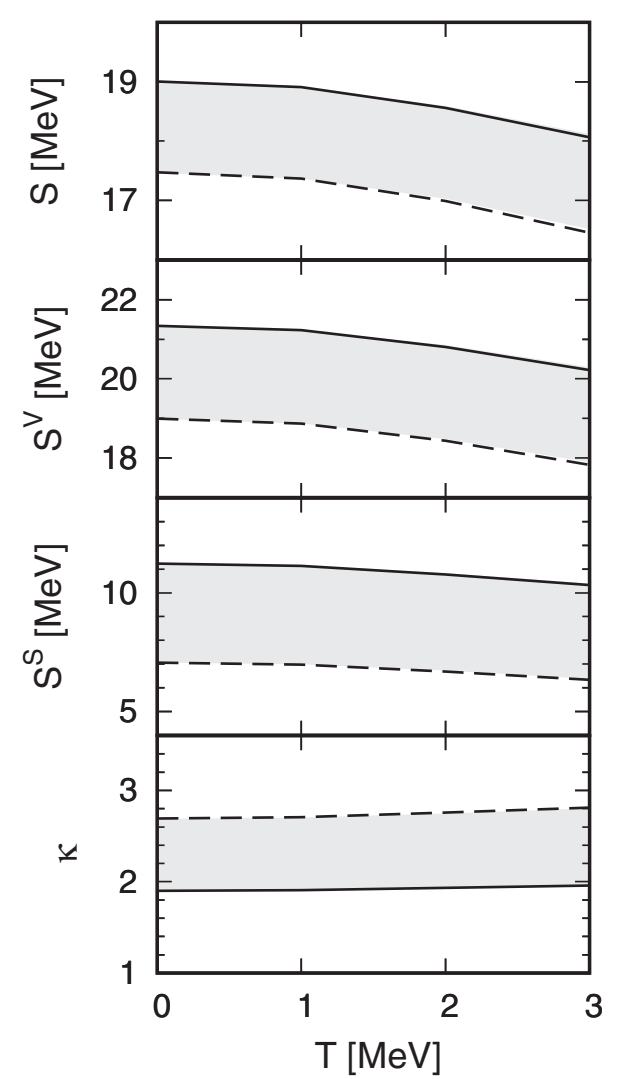

FIG. 12. Same as Fig. 7 (right panel), but with the use of $S_{4}(\rho)$ [Eq. (23)] for values of the parameter $\gamma=0.2$ (solid line) and $\gamma=$ 0.3 (dashed line).

using the density dependence of the symmetry energy $S_{4}(\rho)$ for $\gamma=0.2$ and $\gamma=0.3$ in the case of SLy4 force. This figure corresponds to Fig. 7 (right panel) and one can see the similarities of the presented quantities. As mentioned before, the reason for the latter is the closeness of the corresponding curves at around $\rho / \rho_{0}=0.5$.

Table I reflects the above-noted similarities of the results for $\kappa$ and $S(T)$ at $T=0 \mathrm{MeV}$ in the cases where Eqs. (19) and (23) are used. However, the results obtained for $\kappa$ using the density dependence from Eq. (22) are quite different from those in Table I with either set of parameter values: $\kappa=10.2$ for $S_{1}(\rho), \kappa=11.9$ for $S_{2}(\rho)$, and $\kappa=13.82$ for $S_{3}(\rho)$ [while the values of $S(T)$ for the same symmetry energy forms are $14.62,14.22$, and $13.85 \mathrm{MeV}$, respectively]. The reason for

TABLE I. Values of the parameter $\gamma$, the ratio $\kappa$, and the symmetry energy $S(T)$ (in $\mathrm{MeV}$ ) for the ${ }^{78} \mathrm{Ni}$ nucleus at $T=0 \mathrm{MeV}$ in the case of the density dependence of the symmetry energy given by Eq. (19) (left part) and by $S_{4}(\rho)$ [Eq. (23)] (right part).

\begin{tabular}{lrcccc}
\hline \hline$\gamma$ & $\kappa$ & $S(T)$ & $\gamma$ & $\kappa$ & $S(T)$ \\
\hline 0.3 & 1.66 & 21.35 & 0.2 & 1.90 & 19.00 \\
0.4 & 2.64 & 19.08 & 0.3 & 2.69 & 17.48 \\
0.5 & 4.09 & 17.20 & 0.4 & 3.74 & 16.23 \\
0.7 & 10.67 & 14.26 & 0.7 & 9.82 & 13.58 \\
\hline \hline
\end{tabular}

this comes from the fact that the integrand in Eq. (18) for $\kappa$ is strongly peaked around $\rho\left(R_{1 / 2}\right) / \rho_{0}=0.5$, and the results depend on the behavior of $S_{2}(\rho)$ with respect to $S_{0}(\rho)$ and $S_{4}(\rho)$ in this region (see Fig. 10). It is also worth mentioning that the large values of the ratio $\kappa$ given above are comparable with those presented in Table I at large values of the parameter $\gamma(\gamma=0.7)$.

\section{CONCLUSIONS}

In the present work we perform calculations of the temperature dependence of the NSE $S(T)$, its volume $S^{V}(T)$ and surface $S^{S}(T)$ components, and their ratio $\kappa(T)=$ $S^{V}(T) / S^{S}(T)$. Our method is based on the local density approximation. It uses the coherent density fluctuation model [35,36] with $T$-dependent proton $\rho_{p}(r, T)$, neutron $\rho_{n}(r, T)$, and total $\rho_{\text {total }}(r, T)=\rho_{p}(r, T)+\rho_{n}(r, T)$ density distributions. The latter are calculated within the self-consistent Skyrme HFB method using the cylindrical transformed harmonic-oscillator basis (HFBTHO) [40,41] and the corresponding code with SkM* and SLy4 Skyrme forces. In the calculations we used in Eqs. (15) and (18) different density dependences of the symmetry energy $S[\rho(x, T)]$, namely, Eq. (19) in Sec. III A and the alternative cases, Eqs. (22) and (23), in Sec. III B. The quantities of interest are calculated for the isotopic chains of $\mathrm{Ni}, \mathrm{Sn}$, and $\mathrm{Pb}$ nuclei.

The main results of the present work can be summarized as follows:

(i) With increasing $T$, the quantities $S, S^{V}$, and $S^{S}$ decrease, while $\kappa$ slightly increases for all the isotopes in the three chains both for Skyrme forces and for all used density dependences of the symmetry energy. The same conclusion can be drawn for the thermal evolution of the mentioned quantities in the cases of the three considered double-magic nuclei: ${ }^{78} \mathrm{Ni},{ }^{132} \mathrm{Sn}$, and ${ }^{208} \mathrm{~Pb}$.

(ii) The results for $S(T), S^{V}(T), S^{S}(T)$, and $\kappa(T)$ are sensitive to the choice of the density dependence of the symmetry energy $S[\rho(x, T)]$ in Eqs. (15) and (18). In Sec. III A the sensitivity of the studied quantities to the values of the parameter $\gamma$ in Eq. (19) is shown. In Sec. III B we consider in detail the results when other, different density dependences of the symmetry energy [Eqs. (19), (22), and (23)] are used. The similarities and differences between the results from various functional forms are related to the behavior of the corresponding values of $S[\rho(x, T)]$ around the value of the ratio $\rho(x, T) / \rho_{0}=0.5$ for which the CDFM weight function has a maximum.

(iii) In the cases of the double-magic ${ }^{78} \mathrm{Ni}$ and ${ }^{132} \mathrm{Sn}$ nuclei we observe "kinks" for $T=0 \mathrm{MeV}$ in the curves of $S(T), S^{V}(T), S^{S}(T)$, and $\kappa(T)$ but not in the case of $\mathrm{Pb}$ isotopes. This effect was also observed in our previous works. It is also worth mentioning that the kinks are blurred and eventually disappear as $T$ increases, demonstrating its close relationship with the shell structure. 


\section{ACKNOWLEDGMENTS}

Three of the authors (M.K.G., A.N.A., and D.N.K) are grateful for the support of the Bulgarian Science
Fund under Contract No. DFNI-T02/19. E.M.G. and P.S. acknowledge support from MINECO (Spain) under Contract Nos. FIS2014-51971-P (E.M.G. and P.S.) and FPA201565035-P (E.M.G.).
[1] Topical issue on Nuclear Symmetry Energy, Guest editors: BaoAn Li, Angels Ramos, Giuseppe Verde, and Isaac Vidaña, Eur. Phys. J. A 50, 2 (2014).

[2] J. M. Lattimer and M. Prakash, Phys. Rep. 442, 109 (2007).

[3] B. A. Li et al., Phys. Rep. 464, 113 (2008).

[4] B. K. Agrawal, J. N. De, S. K. Samaddar, M. Centelles, and X. Viñas, Eur. Phys. J. A 50, 19 (2014).

[5] S. K. Samaddar, J. N. De, X. Viñas, and M. Centelles, Phys. Rev. C 76, 041602(R) (2007).

[6] S. K. Samaddar, J. N. De, X. Viñas, and M. Centelles, Phys. Rev. C 78, 034607 (2008).

[7] J. N. De and S. K. Samaddar, Phys. Rev. C 85, 024310 (2012).

[8] M. K. Gaidarov, A. N. Antonov, P. Sarriguren, and E. Moya de Guerra, Phys. Rev. C 84, 034316 (2011).

[9] M. K. Gaidarov, A. N. Antonov, P. Sarriguren, and E. M. de Guerra, Phys. Rev. C 85, 064319 (2012).

[10] M. K. Gaidarov, P. Sarriguren, A. N. Antonov, and E. Moya de Guerra, Phys. Rev. C 89, 064301 (2014).

[11] B.-A. Li, Nucl. Phys. News 27, 7 (2017).

[12] Ch. C. Moustakidis, Phys. Rev. C 76, 025805 (2007).

[13] F. Sammarruca, J. Phys. G 37, 085105 (2010); arXiv:0908.1958 [nucl-th].

[14] B. K. Agrawal, D. Bandyopadhyay, J. N. De, and S. K. Samaddar, Phys. Rev. C 89, 044320 (2014).

[15] Z. W. Zhang, S. S. Bao, J. N. Hu, and H. Shen, Phys. Rev. C 90, 054302 (2014).

[16] S. J. Lee and A. Z. Mekjian, Phys. Rev. C 82, 064319 (2010).

[17] B.-A. Li and L.-W. Chen, Phys. Rev. C 74, 034610 (2006).

[18] A. Z. Mekjian, S. J. Lee, and L. Zamick, Phys. Rev. C 72, 044305 (2005).

[19] J. Xu, L.-W. Chen, B.-A. Li, and H.-R. Ma, Phys. Rev. C 75, 014607 (2007); 77, 014302 (2008); Phys. Lett. B 650, 348 (2007).

[20] Z. H. Li, U. Lombardo, H.-J. Schulze, W. Zuo, L. W. Chen, and H. R. Ma, Phys. Rev. C 74, 047304 (2006).

[21] J. Piekarewicz and M. Centelles, Phys. Rev. C 79, 054311 (2009).

[22] I. Vidaña, C. Providência, A. Polls, and A. Rios, Phys. Rev. C 80, 045806 (2009).

[23] F. Sammarruca and P. Liu, Phys. Rev. C 79, 057301 (2009).

[24] P. Danielewicz, arXiv:1003.4011 [nucl-th].

[25] M. Brack and P. Quentin, Phys. Lett. B 52, 159 (1974); Phys. Scr. A 10, 163 (1974).

[26] M. Brack, C. Guet, and H. K. Håkansson, Phys. Rep. 123, 276 (1985).

[27] E. Suraud, Nucl. Phys. A 462, 109 (1987).

[28] P. Bonche, S. Levit, and D. Vautherin, Nucl. Phys. A 427, 278 (1984); 436, 265 (1985).

[29] J. N. De, N. Rudra, Subrata Pal, and S. K. Samaddar, Phys. Rev. C 53, 780 (1996).

[30] M. Brack, Phys. Rev. Lett. 53, 119 (1984).

[31] E. Khan, N. Van Giai, and N. Sandulescu, Nucl. Phys. A 789, 94 (2007).
[32] N. Sandulescu, Phys. Rev. C 70, 025801 (2004).

[33] C. Monrozeau, J. Margueron, and N. Sandulescu, Phys. Rev. C 75, 065807 (2007).

[34] E. Yüksel, E. Khan, K. Bozkurt, and G. Colò, Eur. Phys. J. A 50, 160 (2014).

[35] A. N. Antonov, V. A. Nikolaev, and I. Zh. Petkov, Bulg. J. Phys. 6, 151 (1979); Z. Phys. A 297, 257 (1980); 304, 239 (1982); Nuovo Cimento A 86, 23 (1985); A. N. Antonov et al., ibid. 102, 1701 (1989); A. N. Antonov, D. N. Kadrev, and P. E. Hodgson, Phys. Rev. C 50, 164 (1994).

[36] A. N. Antonov, P. E. Hodgson, and I. Zh. Petkov, Nucleon Momentum and Density Distributions in Nuclei (Clarendon Press, Oxford, 1988); Nucleon Correlations in Nuclei (SpringerVerlag, Berlin-Heidelberg-New York, 1993).

[37] K. A. Brueckner, J. R. Buchler, S. Jorna, and R. J. Lombard, Phys. Rev. 171, 1188 (1968).

[38] K. A. Brueckner, J. R. Buchler, R. C. Clark, and R. J. Lombard, Phys. Rev. 181, 1543 (1969).

[39] A. N. Antonov, D. N. Kadrev, M. K. Gaidarov, P. Sarriguren, and E. M. de Guerra, Phys. Rev. C 95, 024314 (2017).

[40] M. V. Stoitsov, N. Schunck, M. Kortelainen, N. Michel, H. Nam, E. Olsen, J. Sarich, and S. Wild, Comp. Phys. Comm. 184, 1592 (2013).

[41] M. V. Stoitsov, J. Dobaczewski, W. Nazarewicz, and P. Ring, Comput. Phys. Comm. 167, 43 (2005).

[42] M. V. Stoitsov, I. Zh. Petkov, and E. S. Kryachko, C. R. Bulg. Acad. Sci. 40, 45 (1987); M. V. Stoitsov, Nuovo Cimento A 98, 725 (1987).

[43] E. Feenberg, Rev. Mod. Phys. 19, 239 (1947).

[44] A. G. W. Cameron, Canad. J. Phys. 35, 1021 (1957).

[45] H. A. Bethe, Theory of Nuclear Matter, Annual Review of Nuclear Science Vol. 21 (Annual Reviews, Palo Alto, 1971), Chap. 9, pp. 93-244.

[46] A. E. S. Green, Rev. Mod. Phys. 30, 569 (1958).

[47] W. D. Myers and W. J. Swiatecki, Nucl. Phys. A 81, 1 (1966).

[48] P. Danielewicz, Nucl. Phys. A 727, 233 (2003).

[49] P. Danielewicz, arXiv:nucl-th/0411115.

[50] P. Danielewicz and J. Lee, Nucl. Phys. A 922, 1 (2014), and references therein.

[51] P. Danielewicz and J. Lee, Int. J. Mod. Phys. E 18, 892 (2009).

[52] P. Danielewicz and J. Lee, in IX Latin American Symposium On Nuclear Physics and Applications, edited by R. Alarcon, E. Ayala, C. Granja, and N. Medina, AIP Conf. Proc. 1423 (AIP, Melville, NY, 2012), p. 29.

[53] M. B. Tsang, J. R. Stone, F. Camera, P. Danielewicz, S. Gandolfi, K. Hebeler, C. J. Horowitz, Jenny Lee, W. G. Lynch, Z. Kohley, R. Lemmon, P. Möller, T. Murakami, S. Riordan, X. Roca-Maza, F. Sammarruca, A. W. Steiner, I. Vidaña, and S. J. Yennello, Phys. Rev. C 86, 015803 (2012).

[54] M. B. Tsang, Yingxun Zhang, P. Danielewicz, M. Famiano, Zhuxia Li, W. G. Lynch, and A. W. Steiner, Phys. Rev. Lett. 
102, 122701 (2009); M. B. Tsang et al., Int. J. Mod. Phys. E 19, 1631 (2010).

[55] A. Ono, P. Danielewicz, W. A. Friedman, W. G. Lynch, and M. B. Tsang, Phys. Rev. C 70, 041604(R) (2004).

[56] P. Danielewicz, arXiv:nucl-th/0607030.

[57] M. Warda, X. Viñas, X. Roca-Maza, and M. Centelles, Phys. Rev. C 81, 054309 (2010).

[58] M. Centelles, X. Roca-Maza, X. Viñas, and M. Warda, Phys. Rev. C 82, 054314 (2010).

[59] A. W. Steiner, M. Prakash, J. M. Lattimer, and P. J. Ellis, Phys. Rep. 411, 325 (2005).

[60] P. Danielewicz and J. Lee, Nucl. Phys. A 818, 36 (2009).

[61] A. E. L. Dieperink and P. Van Isacker, Eur. Phys. J. A 32, 11 (2007).

[62] V. M. Kolomietz and A. I. Sanzhur, Eur. Phys. J. A 38, 345 (2008); Phys. Rev. C 81, 024324 (2010).

[63] N. Nikolov, N. Schunck, W. Nazarewicz, M. Bender, and J. Pei, Phys. Rev. C 83, 034305 (2011).

[64] H. Jiang, G. J. Fu, Y. M. Zhao, and A. Arima, Phys. Rev. C 85, 024301 (2012).
[65] M. Chun-Wang et al., Chinese Phys. Lett. 29, 092101 (2012).

[66] J. N. De, S. K. Samaddar, and B. K. Agrawal, Phys. Lett. B 716, 361 (2012).

[67] W. Guo, M. Colonna, V. Greco, U. Lombardo, and H. J. Schultze, arXiv:1804.04827 [nucl-th].

[68] A. N. Antonov, M. K. Gaidarov, P. Sarriguren, and E. Moya de Guerra, Phys. Rev. C 94, 014319 (2016).

[69] N. Wang, M. Liu, L. Ou, and Y. Zhang, Phys. Lett. B 751, 553 (2015).

[70] W. D. Myers and W. J. Swiatecki, Ann. Phys. 55, 395 (1969).

[71] F. Sammarruca, Mod. Phys. Lett. A 32, 1730027 (2017).

[72] A. Akmal, V. R. Pandharipande, and D. G. Ravenhall, Phys. Rev. C 58, 1804 (1998).

[73] P. Russotto et al., Phys. Rev. C 94, 034608 (2016).

[74] J. Dong, W. Zuo, J. Gu, and U. Lombardo, Phys. Rev. C 85, 034308 (2012).

[75] T. Mukhopadhyay and D. N. Basu, Nucl. Phys. A 789, 201 (2007).

[76] B. K. Agrawal, J. N. De, S. K. Samaddar, G. Colò, and A. Sulaksono, Phys. Rev. C 87, 051306(R) (2013). 